\title{
Comparison of LSB and Predictive Coding using PSNR and MSE
}

\author{
Jagreeti Kaur \\ Department of Computer Science \& Engineering \\ Baba Banda Singh Bhadur Engineering College, \\ Fatehgarh Sahib, Punjab, India
}

\author{
Baljit Singh, Ph.D \\ Department of Computer Science \& Engineering \\ Baba Banda Singh Bhadur Engineering College \\ Fatehgarh Sahib, Punjab, India
}

\begin{abstract}
In the era of internet, in every communication images play a very important role. Images are used for secret communication also like for military purposes, hiding the serial number or copyright also to embed the virus and other bad things etc. To hide data in image in such a way that the existence of the message is unknown is steganography. Different techniques are used to hide data in the image to achieve high quality of stego image and high embedding capacity. In this paper, two steganographic techniques re used i.e. LSB and predictive coding. The stego image which is formed by both the techniques are then compared using two parameters i.e. PSNR and MSE
\end{abstract}

\section{Keywords}

Predictive Coding, Least Significant Bit(LSB), Steganography, MSE, PSNR.

\section{INTRODUCTION}

The present age the age of internet. Everyone use internet to get and send information from one point to another. Internet has become the fastest mean to send and receive the data. In today's era, without internet life seems to be stop. Images play a vital role in communication on internet. Images are also used for secret communication. The technique of doing secret communication is known as steganography. Steganography is the art and science of invisible communications. This can be done by hiding data in other data [1].

Steganography is derived from the greek word 'Steganos' which means covered or hidden and 'Graphy' means writing.

Greeks use different techniques to send the secret message to desired destination. The concepts of invisible ink, punching the message on slaves head, wax table, biliterals are used by Greeks [2]. With the advancement of technology new concepts used to hide the secret data in images.

In steganography the secret data is hidden in covered image. The covered image with secret data is known as Stego-image.

Image Steganography is performed in two steps. To embed the secret message in the covered image and the resultant image is the stego image is the embedding process. Secondly, the extraction of secret message from the stego-image is the extraction process.

The images are of two types. Reversible image is the first type of image in which the quality of the image will remain same as the host image or covered image after extraction. The second type is irreversible image, the quality of image will get under distortion after the extraction of the secret message from the stego-image. The images are also of two formats i.e. raw format and compressed format. The raw format is where the image size is large and compressed format is where the image is compressed and of small size than raw format. As the bandwidth is needed to transmit an image through internet, so the compressed format is used [4].

\section{LITERATURE SURVEY}

The oldest technique to hide secret data is Least Significant Bit (LSB) in image steganography. In LSB, the secret data is hidden in the bits of pixels of an image. The image will not get under distortion after the embedding secret data using LSB. In case of 24-bit image, the 3 bits of each pixels are used to embed secret data.

Viji et al. [5] proposed an algorithm to hide large amount of secret data into color images. The algorithm is based on adaptive image filtering and adaptive image segmentation. The embedding size of secret message is large because of the availability of more numbers of bits in color image.

Majumder et al. [6] gives an algorithm to hide large quantity of data in color BMP images. The algorithm is use to hide any input data in BMP image which have 24-bits, each is separated by 4-bits. The 2 left bits contains the highest value and the 2 right bits contain the lowest value. The algorithm works only on BMP color images.

Sivaram et al. [7] estimate the secret message in the image. Random pixels are used to embed the secret message into two positions of LSB. If 2 bits used to hide data in LSB technique, the large content of data is possible to hide.

Predictive coding is used for image and text compression. The differencing value of current data estimation and actual current data encodes the predictive coding. The concept of Median Edge Detector (MED) is used in predictive coding. for each image pixel the predictor values are generated by MED predictor. The predictor values that are generated by MED predictor is called predictive image. Basically, MED predictor is used as a substitute of the difference between the adjacent pixels [8].Error values are obtained as resultant of differencing the original and predictive image. The error values than used in entropy coding stage.

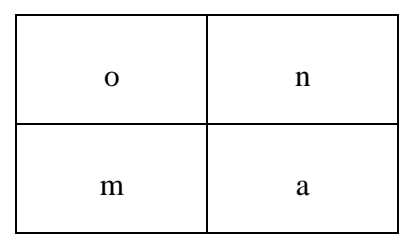

Fig 1: Predictive Template 
The neighboring pixels are used to generate the predictive image in fig.1. Let a be the current predictive pixel having neighbors as $\mathrm{m}, \mathrm{n}$, o. In order to detect the vertical and horizontal edges, MED predictor uses the past data i.e. $\mathrm{m}, \mathrm{n}, \mathrm{o}$ in the predictive template. Let the vertical edge on left side of $\mathrm{a}$, the MED predictor use $\mathrm{N}$ as the predictive value. When the upper side of a is act as horizontal edge, the MED predictor uses $\mathrm{M}$ as the predictive value. If no edge appears in template, then MED predictor use $\mathrm{M}+\mathrm{N}-\mathrm{O}$ as the predictive value.

In the extracting stage, the error values which are obtained from entropy coding stage are used and predictive image also generate the same MED predictor. The addition of the predictive image and the error values results as the Original image [4].

Yuan et al. [9] propose a data hiding scheme which is based on prediction that embeds secret data into compression codes during image compression. The scheme is divided into two stages. Predictive stage and an entropy coding stages are the two stages used by Yaun. Yang et al. [10] proposed scheme based on hiding data into edge areas than smooth areas in the host image, which does not differentiate textures from edges and causes serious degradation in actual edge areas. Tsai et al. [11] used the scheme for reversible data hiding is linear prediction for medical images. The scheme is based on histogram shifting which is limited by the hiding capacity. Hossain et al. [12] proposed three different steganographic methods for gray level images. The methods are four neighbours, diagonal neighbours and eight neighbours methods.

\section{IMAGE QUALITY PARAMETERS}

The degree of distortion of image can be measured by using mean square error (MSE) and peak signal-to-noise ratio (PSNR). Both MSE and PSNR are used because they represent the grey value error of the whole image. All the pixels of an image are equally important. With the use of PSNR or MSE, gray-value difference between corresponding pixels of the original image and the pixels of distorted image are considered. All the pixels of an image are independent of their neighbor pixels. Therefore, pixels at different position have different effect on human visual system (HVS) [15].

Mean Square Error could be estimated in one of numerous approaches to quantify the contrast between values implied by an evaluation and correct quality being certified.

$$
M S E=\frac{1}{m^{*} n} \sum_{i=0}^{m-1} \sum_{i=0}^{n-1}[I(i, j)-K(i, j)]^{2}
$$

Where the size of image is $m x n$

There are numerous measures for examining image quality, such as, the mean structural similarity, mean absolute error, mean square error (MSE), and peak signal-to-noise ratio (PSNR). It is processed by averaging the squared intensity differences of distorted and original image pixels, as well as the related amount of the PSNR.

In the most recent decade, much exertion has gone into the advancement of image quality measures that advantage of well-known characteristics of the human vision framework (HVS). Starting from these truths, the proposed color image quality measure (CQM) takes after another methodology of changing the usage system for the PSNR [13].

PSNR is expressed in terms of the logarithmic decibel scale because many signals have a very wide dynamic range. The
PSNR is used as a measure of quality of reconstruction of lossy compression .In the case of compression, the signal is the original data, and the noise is the error.

In some cases, reconstruction may appear to be closer to the original than another, even though it has a lower PSNR (a higher PSNR would normally indicate that the reconstruction is of higher quality) [14].

PSNR is defined as:

$$
\begin{aligned}
& P S N R=10 \log _{10}\left(\frac{M A X^{2}}{M S E}\right) \\
& =20 \log _{10}\left(\frac{M A X}{\sqrt{M S E}}\right) \\
& =20 \log _{10}(M A X)-10 \log _{10}(M S E)
\end{aligned}
$$

Here, MAX is the maximum possible pixel value of the image. When the pixels are represented using 8 bits per sample, this is 255. Typical values for the PSNR in lossy image and video compression are between 30 and $50 \mathrm{~dB}$, where higher is better. Acceptable values for wireless transmission quality loss are considered to be about $20 \mathrm{~dB}$ to $25 \mathrm{~dB}$. When the two images are identical, the MSE will be zero.

\section{PROPOSED METHOD}

LSB Algorithm

i. Input covered or host image as $\mathrm{C}(\mathrm{x}, \mathrm{y})$

ii. Find the size of the image $\mathrm{C}$ i.e. $\mathrm{MxN}$

iii. Choose the text to hide in image $\mathrm{C}$ and store in constant $\mathrm{Y}$

iv. Apply

$\mathrm{S}=$ uint8(bitor(bitand(C,bitcmp $\left.\left(2^{\mathrm{n}-1}, 8\right)\right)$, $\operatorname{bitshift}(Y, n-$ 8))) and $\mathrm{E}=\operatorname{uint} 8(\operatorname{bitand}(255, \operatorname{bitshift}(\mathrm{S}, 8-\mathrm{n})))$

v. Find the error value by substracting host image and stego image

vi. Close the text file.

Predictive Coding Algorithm

i. Input Source Image as $\mathrm{C}(\mathrm{x}, \mathrm{y})$

ii. Calculate the size of the Image I i.e. $\mathrm{m} \times \mathrm{n}$

iii. Choose the text to hide in the image $\mathrm{C}$

iv. Stego Image be as SI

v. $\operatorname{SI}(1: \mathrm{m}, 1)=\mathrm{C}(1: \mathrm{m}, 1)$

$\mathrm{SI}(1, \mathrm{i}: \mathrm{n})=\mathrm{C}(1,1: \mathrm{n})$

$\mathrm{SI}(1: \mathrm{m}, \mathrm{n})=\mathrm{C}(1: \mathrm{m}, \mathrm{n})$

$\mathrm{SI}(\mathrm{m}, 1: \mathrm{n})=\mathrm{C}(\mathrm{m}, 1: \mathrm{n})$

vi. for $\mathrm{k}=2: \mathrm{m}-1$

for $1=2: n-1$

vii. Apply predictive coding formula

$\mathrm{SI}(\mathrm{k}, \mathrm{l})=\mathrm{C}(\mathrm{k}, \mathrm{l})-(\mathrm{C}(\mathrm{k}, \mathrm{l}-1) / 2+\mathrm{C}(\mathrm{k}-1, \mathrm{l}) / 4+\mathrm{C}(\mathrm{k}-1, \mathrm{l}-1) / 8+\mathrm{C}(\mathrm{k}-$ $1,1+1) / 8)$

viii. $\operatorname{SI}(1: \mathrm{m}, 1)=\mathrm{C}(1: \mathrm{m}, 1)$

$\mathrm{SI}(1 ; \mathrm{m}, \mathrm{n})=\mathrm{C}(1,1: \mathrm{n})$

$\mathrm{SI}(1: \mathrm{m}, \mathrm{n})=\mathrm{C}(1: \mathrm{m}, \mathrm{n})$

$\mathrm{SI}(\mathrm{m}, 1: \mathrm{n})=\mathrm{C}(\mathrm{m}, 1: \mathrm{n})$

ix. for $\mathrm{k}=2: \mathrm{m}-1$

for $1=2: n-1$

$\mathrm{x}$. Apply predictive coding formula again for this loop

$\mathrm{SI}(\mathrm{k}, \mathrm{l})=\mathrm{C}(\mathrm{k}, \mathrm{l})+(\mathrm{SI}(\mathrm{k}, 1-1) / 2+\mathrm{SI}(\mathrm{k}-1,1) / 4+\mathrm{SI}(\mathrm{k}-1,1-$

1)/8+SI(k-1,1+1)/8);

xi. Close the text file. 


\section{EXPERIMENTAL RESULTS}

The section contains experimental results of the images as under using two parameters i.e. PSNR and MSE.

The text file which is used to hide in image is in .txt format and size of the file is $92.8 \mathrm{~KB}$
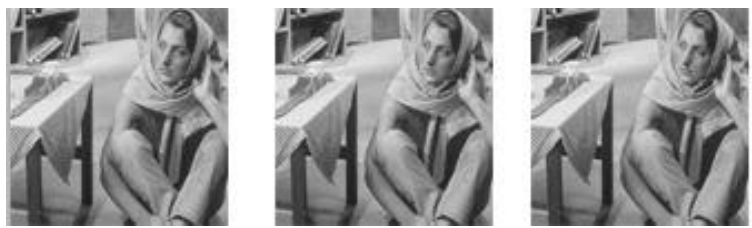

Fig 2(i) : Source Image of Barbara.png, Fig 2(ii): Stego Image of Barbara.png using LSB, Fig 2(iii): Stego Image of Barbara.png using Predictive Coding
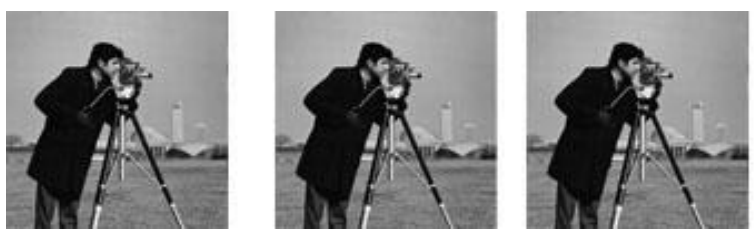

Fig 3(i) : Source Image of Cameraman.gif, Fig 3(ii): Stego Image of Cameraman.gif using LSB, Fig 3(iii): Stego Image of Cameraman.gif using Predictive Coding
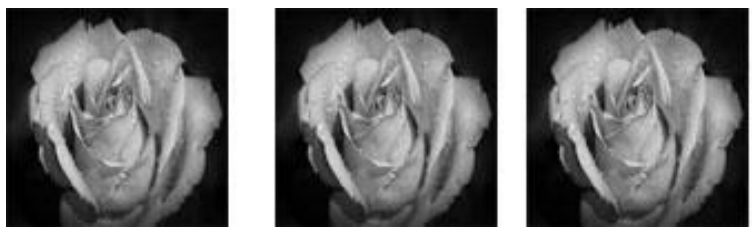

Fig 4(i) : Source Image of Flower.jpg, Fig 4(ii): Stego Image of Flower.jpg using LSB, Fig 4(iii): Stego Image of Flower.jpg using Predictive Coding
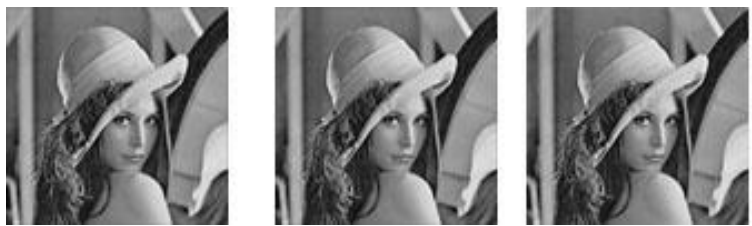

Fig 5(i): Source Image of Lena.jpg, Fig 5(ii): Stego Image of Lena.jpg using LSB, Fig 5(iii): Stego Image of Lena.jpg using Predictive Coding
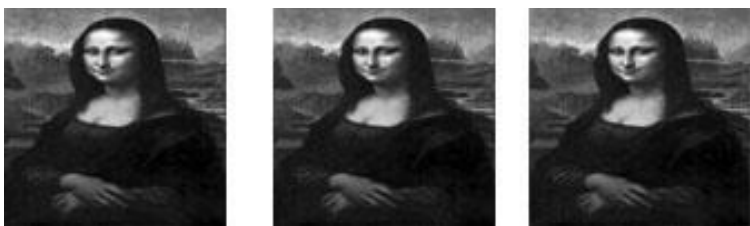

Fig 6(i): Source Image of Mona_lisa.png, Fig 6(ii): Stego Image of Mona_lisa.png using LSB, Fig 6(iii): Stego Image of Mona_liisa.png using Predictive Coding
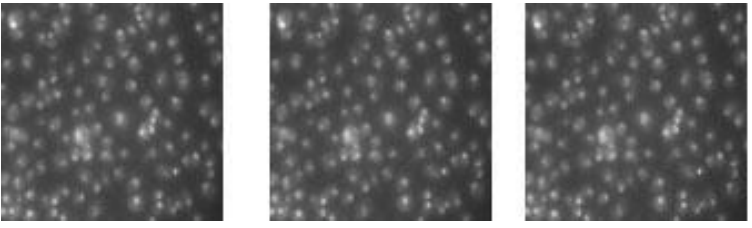

Fig 7(i): Source Image of Nuclei.png, Fig 7(ii): Stego Image of Nuclei.png using LSB, Fig 7(iii): Stego Image of Nuclei.png using Predictive Coding
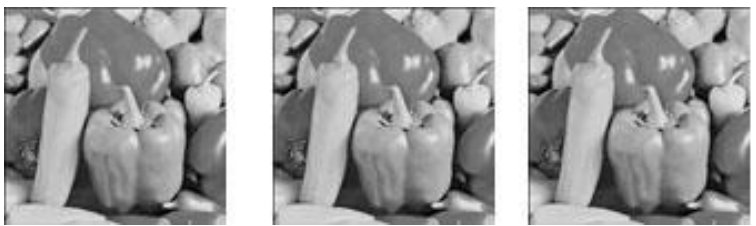

Fig 8(i): Source Image of Peppers.png, Fig 8(ii): Stego Image of Peppers.png using LSB, Fig 8(iii): Stego Image of Peppers.png using Predictive Coding
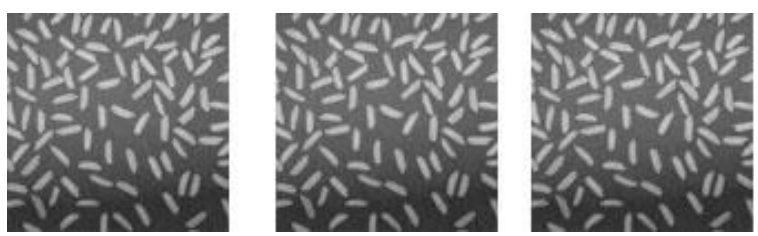

Fig 9(i): Source Image of Rice.gif, Fig 9(ii): Stego Image of Rice.gif using LSB, Fig 9(iii): Stego Image of Rice.gif using Predictive Coding

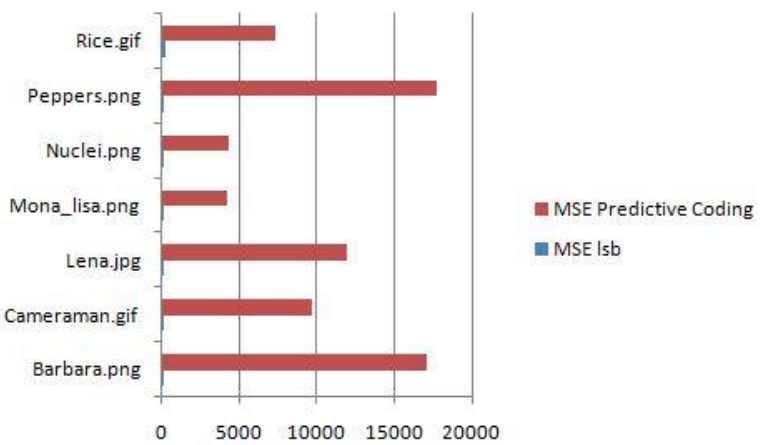

Fig 10: Variation of MSE in Stego-images of Predictive Coding Technique and Stego-images of LSB Technique

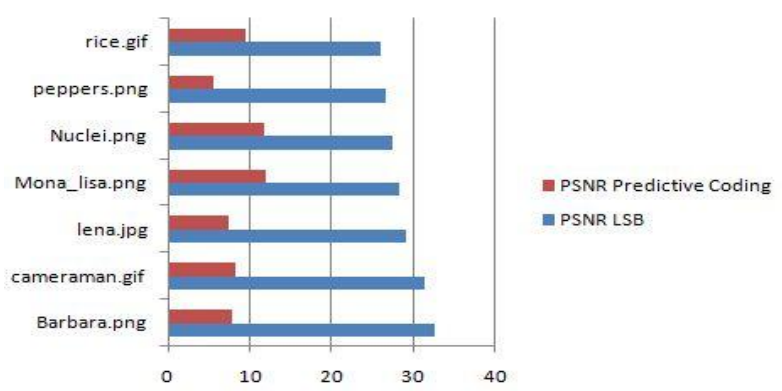

Fig 11: Variation of PSNR in Stego-images of Predictive Coding Technique and Stego-images of LSB Technique 


\section{CONCLUSION}

Image Steganography is used for the secure transmission of data which is hidden in the image. The parameters are used to check the quality of the stego image are MSE and PSNR. LSB can get change if intruder get know about the LSB bit and can corrupt the data. Here the experimental results show high PSNR and low MSE is for good quality of stego image but not for secure transmission. For the secure transmission, Predictive coding is used. In predictive coding, data cannot get corrupted and intruder will not get notice about the hidden data. Predictive coding also results as reversible image.

\section{ACKNOWLEDGMENTS}

Our thanks to the experts who have contributed towards development of the template.

\section{REFERENCES}

[1] Khare Akhil, Kumari Meenu, Khare Pallavi, 2010"Efficient Algorithm for Digital Image Steganography", Journal of Information, Knowledge and Research in Computer Science and Applications, Vol.1, No. 1,pp. 1-5

[2] Streetman Kibbee D., "Steganography Art of Covert Communication"

[3] Wu Hsien-Chu, Wang Hua-Cheng, Tsai Chwei-Shyong, Wang Chung-Ming, 2010, "Reversible Image Steganographic Scheme Via Predictive Coding" ,Displays Elsevier, Vol. 31, No. 1, pp. 35-43.

[4] Chugh Gunjan, 2013, "Image Steganography Techniques: A Review Article", ACTA Technica Corviniensis-Bulletin of Engineering, Vol. 6, No. 3, pp. 97-104.

[5] Viji G., Balamurugan J., 2011, “ LSB Steganography in Color and Grayscale Images without using the Transformation", Bonfring International Journal of Advances in Image Processing, Vol. 1, Special issue, pp. 11-14.

[6] Majumder Jayeeta, Mangal Sweta, 2012, "An Overview of Image Steganography using LSB Technique", National Conference on Advances in Computer Science and Applications with International Journal of Computer Applications, pp.1-13.

[7] Sivaram M., DurgaDEvi B., Anne Steffi J., 2012, "Steganography of Two LSB Bits", International Journal of Communication and Engineering, Vol.1, No.1, pp. 8287.

[8] Deepthi Satharla Raga, Devanna H., Sudhakar K., 2013,"An Eminent Reversible Data Hiding System With Edge Prediction and Variation Expansion", International Journal of Computer and Electronics Research, Vol. 2, No. 3, pp. 179-182.

[9] Yu Yuan-Hui, Chang Chin-Chen, $\mathrm{Hu}$ Yu-Chen, 2005,"Hiding Secret Data in Images Via Predictive Coding", Journal of Pattern Reorganization, Vol. 38, No. 5, pp 691-705.

[10] Yang Hengfu, Sun Xingming, Guang Sun, 2009, "A High-Capacity Image Data Hiding Scheme Using Adaptive LSB Substitution", Journal of Radio Engineering, Vol. 18, No. 4, pp. 509-516.

[11] Tsai Piyu, Hu Yu-Chen, Yeh Hsiu- Lein, 2009 , "Reversible Image Hiding Scheme Using Predictive Coding And Histogram Shifting", Journal of Signal Processing, Vol. 89, No. 6, pp. 1129-1143.

[12] Hossain Moazzam, Haque Sasia Al, Sharmin Farhana, 2010, "Variable Rate Steganography in Gray Scale Digital Images Using Neighbourhood Pixel Information", The International Journal of Information Technology, Vol. 7, No. 1, pp.34-38.

[13] Yıldıray Yalman, Ismail Erturk, 2013,“A New Color Image Quality Measure Based On YUV Transformation And PSNR For Human Vision System", Turkish Journal of Electrical Engineering \& Computer Sciences ,Vol. 21, No. 2, pp. 603-612.

[14] http://multimediatechnology.googlecode.com/svnhistor y/r7/trunk/lab2_jpeg/info/PSNR.pdf

[15] Kaiwen Zhang, Shuozhong Wang, and Xinpen Zhang, 2002, "A New Metric for Quality Assessment of Digital Images Based on Weighted-Mean Square Error", Proceedings of SPIE, vol. 4875, No. 1, pp. 552-560. 